\title{
4th European Conference on Schizophrenia Research: together for better treatment and care
}

Dear Colleagues,

In recent years a growing body of cutting-edge schizophrenia research was performed and new evidence was generated with regard to aetiology, symptomatology, treatment and care of schizophrenia. Treatment research on new pharmacological targets and novel mechanisms of action demonstrated encouraging efficacy across a range of symptoms allowing for a better and personalized treatment. New imaging techniques were developed to gain new insights in the pathophysiology of this disorder. Some recent findings may help to diagnose schizophrenia earlier in the course and to predict treatment response. Yet we are still far away from understanding the underlying causes and complexity of schizophrenia corresponding to heterogeneous patient needs. Publication of the fifth edition of the Diagnostic and Statistical Manual of Mental Disorder (DSM-5) earlier this year has led to an intense and controversial discussion beyond the psychiatric community becoming a matter of public interest and media coverage.

The scientific programme of the European Conference on Schizophrenia Research (ECSR) critically discuss recent scientific advances, deals with current controversies, and updates the scientific knowledge about schizophrenia and translate it into clinical practice. The need to have a forum for scientific exchange and to address clinical challenges encouraged, in 2007, the Competence Network on Schizophrenia to initiate this biennial European schizophrenia congress which will be held for the fourth time.

According to this year's motto "Together for better treatment and care", the scientific programme of the ECSR provides a comprehensive overview on recent research projects and developments in schizophrenia treatment. Treatment of negative symptoms, deficits in social cognition, non-adherence, and the promotion and implementation of evidence-based strategies to ensure standards of care as well as basic research topics will be addressed by high quality contributions from acknowledged experts, young scientists, and clinicians. This abstract supplement documents the broad range of topics to be presented in plenary lectures, (prearranged and submitted) symposia, workshops, oral presentations and poster sessions.

As in previous years, the co-organising psychiatric associations and their respective schizophrenia sections play an important role in this joint thematic conference. On occasion of the third ECSR the European Scientific Association on Schizophrenia and other Psychoses (ESAS) took over the responsibility to organise future ECSRs. Yet, the tasks of ESAS are certainly not limited to organising this conference; further tasks may be the identification of emerging issues for schizophrenia research and, as a consequence, outlining a research agenda and initiation of collaborations and networking activities.

On behalf of the Scientific Committee and the organisers, I cordially invite you to participate in the 4th European Schizophrenia Research Conference. It's a pleasure to welcome you in Berlin!

Sincerely yours,

Wolfgang Gaebel

Congress President

President European Scientific Association on Schizophrenia and other Psychoses

Speaker of the Competence Network on Schizophrenia 